\title{
A Sensitive PCR-Based Method for Somatic Mutations Enrichment and Screening
}

\section{Yaming Xiong \\ Hailing Tang}

Department of Thoracic Surgery, The First Affiliated Hospital of Guangzhou Medical University, Guangzhou, 5I0I20, People's Republic of China
Correspondence: Yaming Xiong Email xym02I4@I63.com
Background: EGFR and KRAS are the most frequently mutated genes in lung cancers, occurring in about $60 \%$ of all cases. Mutation genes assay has emerged as a promising bloodbased biomarker for monitoring cancer dynamics noninvasively. However, detection can be challenging in patients where plasma often contains low levels of tumor-derived DNA fragments.

Methods: We have developed a nuclease-based enrichment assay for detecting mutant alleles. The procedure is based on Surveyor endonuclease cleaves mismatched DNA molecules, and these DNA fragments were enriched for mutation screening. We screened lung cancer specimens for mutations in exons 18 and 21 of EGFR, and the majority of activating mutations in lung cancer occur in codons 12 (G12X) and 13 (G13X) of exon 2 of the KRAS gene. The method screened all mutant genes with the same pair primers and three relevant TaqMan probes.

Results: The method can effectively remove wild-type sequences and enrich mutation DNA, and the sensitivity detectable mutant allele frequencies (MAF) achieved $0.001 \%$. The method increases the sensitivity and efficiency of mutation DNA for cancers screening. This highlights the importance of complex DNA variation like mutations in exon 21 of EGFR and exon 2 of the KRAS gene detected by the same probe.

Conclusion: We developed a simple and sensitive methodology for mutation gene screening. The method is a cost-effective and sensitive method for mutation DNA enrichment and detection.

Keywords: liquid biopsy, Surveyor, enrichment, lung cancer

\section{Introduction}

With the advent of personalized medicine, molecular genetic testing has become one of the most valuable diagnostic tools to guide the treatment of cancer patients. ${ }^{1-4}$ In order to be effective, the test needs to accurately detect the genotype of tumor cells with high sensitivity. In the case of somatic mutation detection, samples are usually heterogeneous with low-frequency mutant DNA, and there is usually only one base mutation in the rich wild-type DNA background. ${ }^{5}$ Therefore, somatic mutation detection must have sufficient specificity to distinguish a base difference in heterogeneous alleles and sufficient sensitivity to detect low-level mutations in samples. Various PCR-based detection platforms have been developed for somatic mutation detection, including quantitative PCR and droplet digital PCR. ${ }^{6-11}$ All PCR tests mentioned above have designed a fluorescent dye-labeled mutation site probe and a pair of primers for the targeted amplification sequence. This PCR-based detection will reduce the detection sensitivity of amplification signals that are not needed to 
produce a large amount of wild-type DNA. In many cases, this PCR-based screening adds excellent complexity to the analysis development process because new probe molecules of the target of interest need to be designed and tested in each assay.

We developed a mismatch-specific DNA endonuclease method, Surveyor, scanning for known and unknown mutations in heteroduplex DNA. Surveyor nuclease is an endonuclease that cleaves DNA with high specificity at sites of base-substitution mismatch and other distortions. ${ }^{12}$ These DNA endonucleases cut both strands of a DNA heteroduplex on the $3^{\prime}$ side of the mismatch site. Insertion/deletion mismatches and all base-substitution mismatches are recognized. ${ }^{12}$ Surveyor nuclease is a robust and reproducible tool for mutation detection. ${ }^{12-14}$ It has been used to accurately detect various mutations in human, mammalian, bacterial, and plant genomes. ${ }^{15-18}$

The study developed a new, highly sensitive, and costeffective method for identifying and enriching mutant DNA. Using this method, we genotyped two majorities of activating mutations in lung cancer that occur in EGFR and KRAS genes.

\section{Materials and Methods}

\section{Preparing Hybridized DNA Population}

The hybridized DNA samples can be generated by denaturing and anneal DNA in a thermocycler. Test samples were placed in a $0.2 \mathrm{~mL}$ tube. Place the tube in a thermocycler and run the program following the instruction of the Surveyor enzyme.

\section{Blocking 3' Ends with a Phosphorylation Adaptor}

The adaptor of reverse sequence's 3' end modification with phosphorylation will add to hybridized DNA with TA ligation. The adaptor generated by two complementary oligos (SM1 and SM2) annealed to double-stranded oligos, and the reverse oligo's $3^{\prime}$ end modification with phosphorylation for blocking free 3' end extension. Double-stranded oligos were purified and then ligated to hybridized DNA in the presence of T4 ligase.

\section{Mismatch Digestion}

After adding the adaptor, the hybridized DNA was digested with Surveyor nuclease incubate at $42^{\circ} \mathrm{C}$ for 60 min. Surveyor nuclease is an endonuclease that cleaves DNA at sites of base-substitution mismatch.

\section{Free 3' End Extension with Biotin-Labeled dCTP}

Surveyor nuclease digestion generated the newly $3^{\prime}$ ends were extended with biotin-labeled $\mathrm{dCTP}$ and terminal transferase (TdT). TdT is a template-independent polymerase that catalyzes the addition of deoxynucleotides to the 3' hydroxyl terminus of DNA molecules; these nucleotides subsequently serve as "tails" for cloning.

\section{Add Adaptor with the Newly Generated dCTP "Tails"}

The adaptor was ligated to dCTP "tails" in the presence of T4 ligase. The adaptor generated by two complementary oligos (SM3 and SM4) annealed to double-stranded oligos, the purified double-stranded oligos were ligated with dCTP "tails" by the reaction of dCTP and dGTP.

\section{Enrichment DNA Fragments with Streptavidin Magnetic Beads}

Because streptavidin has a very high affinity for biotin, the fragments contain biotinylated dCTP were pulldown by streptavidin magnetic beads. $50 \mu \mathrm{L}$ streptavidin beads were washed twice with buffer and then added the DNA solution, and the reaction was incubated for one hour at room temperature. The beads were washed three times with buffer and dissolved in sterilized water for PCR screening.

\section{Results}

\section{Experimental Description}

We aimed to develop an enrichment scheme based on Surveyor endonuclease, which can be used to efficiently enrich EGFR and KRAS mutations with low fractional abundance from high background wild-type DNA. In addition, we used standard samples with known mutation frequency to verify the enrichment effect of mutant DNA. The workflow includes mutation sequence enrichment based on heterozygous molecular digestion guiding wild-type sequence elimination (Figure 1). To achieve this enrichment, heterohybrid molecules were digested using Surveyor, resulting in dsDNA molecules with a free $3^{\prime}$ end. ${ }^{12,19,20}$ After digestion, mutant sequences were labeled with biotin-dCTP, and then biotinylated fragments are enriched by streptavidin magnetic beads. 


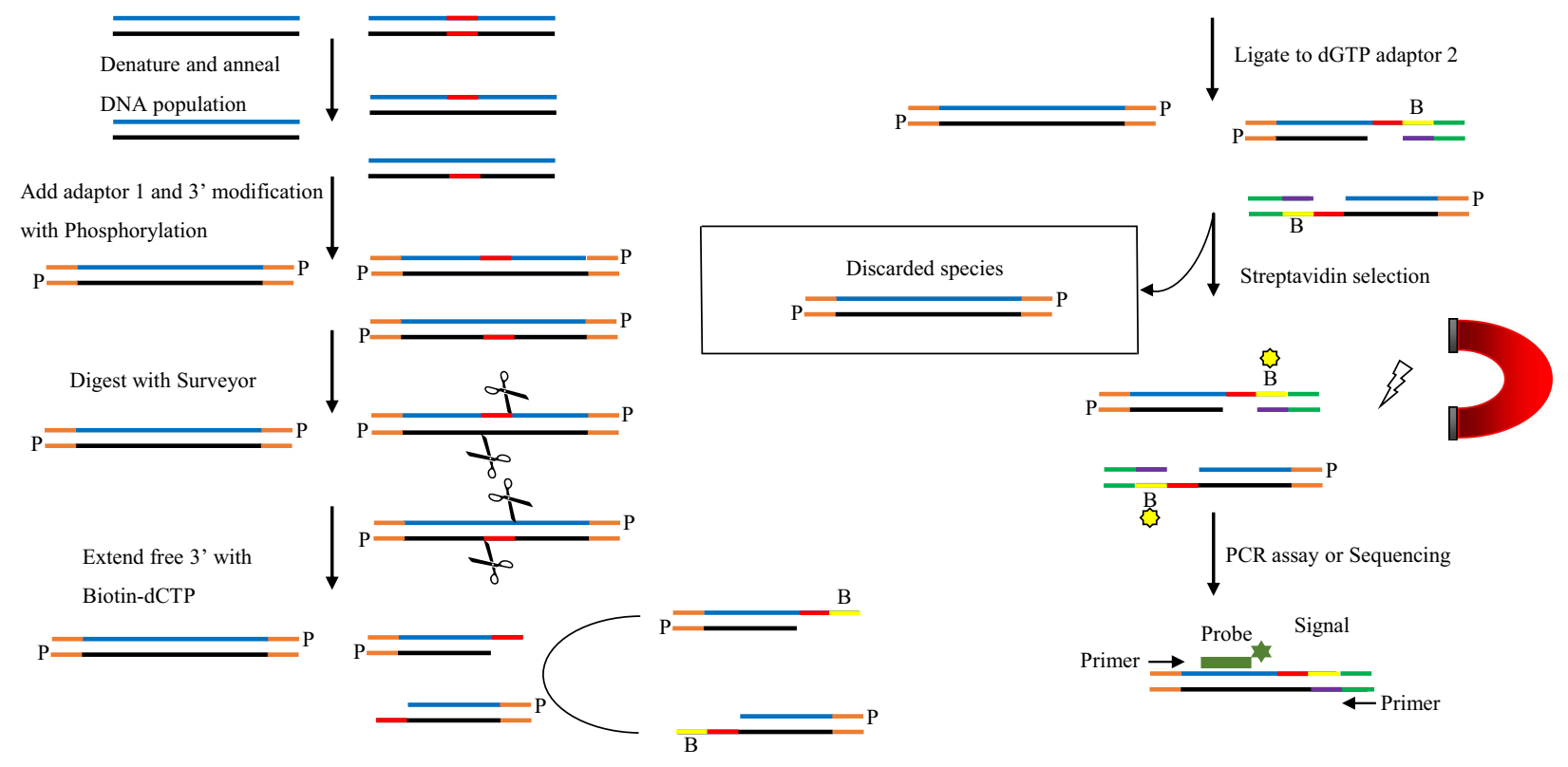

Figure I Surveyor method screening mutant DNA. Hybrid DNA fragments are generated by thermal melting and reannealing. The reannealed products were added adaptors to the $3^{\prime}$ ends of modification with phosphorylation and then digested with Surveyor nuclease. The digested products were tagged with a biotinylated nucleotide for subsequent selection. New adaptors are ligated to free $3^{\prime}$ ends, and then biotinylated fragments are captured using streptavidin magnetic beads. Finally, enriched fragments are used for PCR assay.

\section{Surveyor Method Application to Mutant DNA Enrichment}

We applied it to a known sequence with defined mutation sites to test the Surveyor method. In the initial stage of the study, including optimizing the nuclease enrichment method, droplet digital PCR was used to evaluate the enrichment effect of mutant sequences. In order to evaluate the mutant allele enrichment that can be achieved, we used the reference standard cfDNA (rs-cfDNA) from GeneWell company (China, Shenzhen), which are known to carry the most common hot spot mutations of EGFR and KRAS genes-L858R, 19 deletion, G12D and G13D, respectively. A twofold gradient series of diluted rscfDNA were produced as the template, and the copy number of the enriched fragments was calculated using digital PCR (Figure 2). With the increase in dilution times, the copy number of DNA decreased proportionally. This highlights the excellent analytical reliability of the Surveyor method in enriching cancer mutant DNA.

In order to verify that the Surveyor method can completely remove the wild-type gene sequences, we designed a drop-off method to verify this effect (Figure 3A). The method assigns two signal probes to the wild-type DNA sequence. Drop-off probes in FAM show that the signal produced by a wild-type allele will

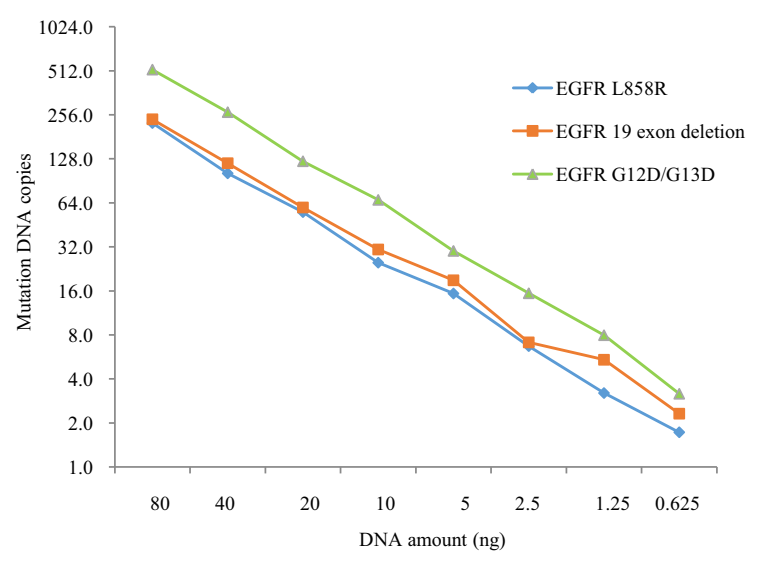

Figure 2 ddPCR measurements of mutation DNA copy numbers across serial dilution cfDNA samples.

always be positive for both FAM and VIC (Figure 3B). Surveyor method enriched DNA and the signal of VIC will be positive. The FAM signal will be negative for the target sequence complementary to the probe digested by the Surveyor enzyme. We screened eight samples with EGFR L858R mutation, and the FAM signal was not detected in all assays (Figure 3C). The result showed that the Surveyor method could effectively remove wild-type sequences and enrich mutant DNA. 
A

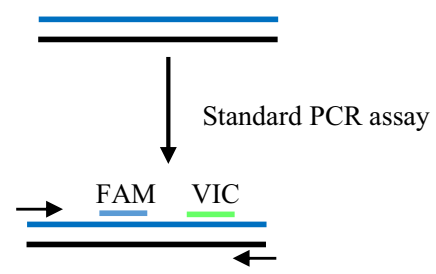

B

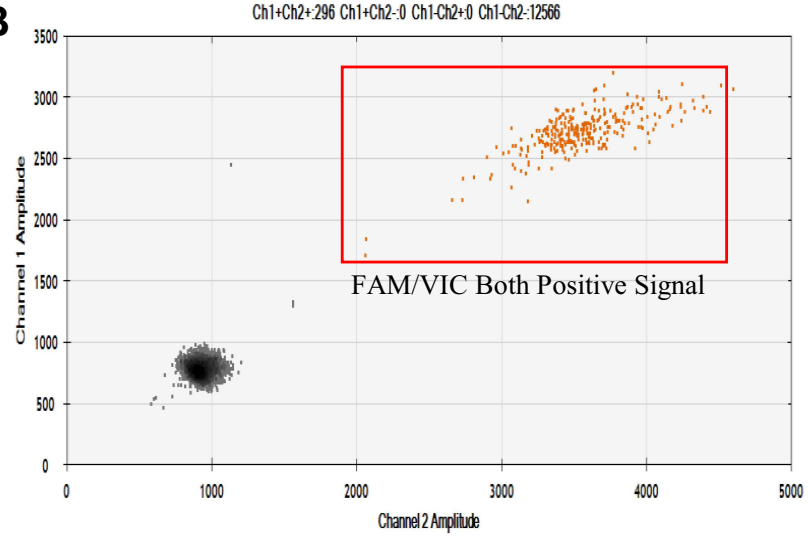

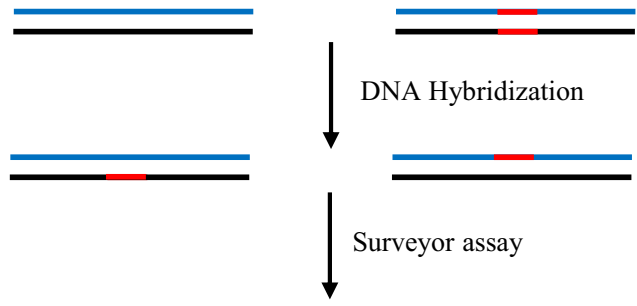

FAM No Signal

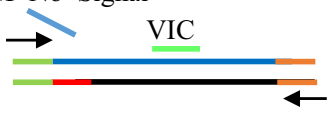

C. Ch1 $1+$ Ch2 $2+0$ Ch1+Ch2:-0 Ch1-Ch2 $2+40$ Ch1 1 -Ch2 $2: 13026$

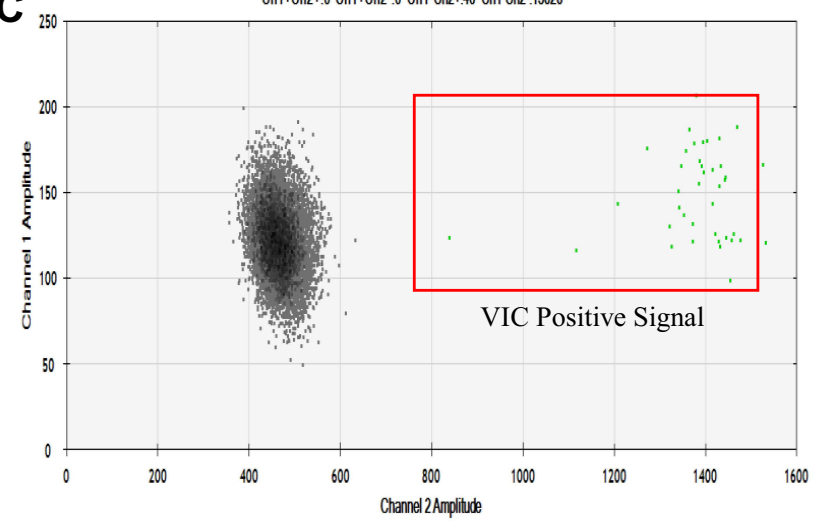

Figure 3 Drop-off experiment set up for analysis mutant DNA enrichment result of Surveyor method. (A) Two configurations of drop-off assays are possible and are shown above, assign a signal of VIC to wild-type and FAM to mutant alleles. The signal produced by wild-type sequences will always be positive for both FAM and VIC, while only the signal VIC made in the Surveyor method enriched mutant DNA sequences. (B) The signal positive for both FAM and VIC in wild-type sequences. (C) The VIC signal positive and FAM negative in Surveyor method enriched mutant DNA sequences.

\section{Surveyor Method Changes Copies Number of Detected Mutant DNA}

When there are multiple mutant DNA copies, DNA can be reliably detected in plasma. However, when the number of mutant DNA is low, the analysis of a single base mutation site may produce negative results even if the analysis with perfect analytical sensitivity is used due to sampling noise. ${ }^{21}$ In the study, mutant genes were hybridized with wild-type sequences, and Surveyor digested mismatched sequences and generated more fragments for subsequent mutant DNA screening. Under the treatment of the Surveyor method, one copy of the mutant gene will become four copies of the subsequent assay. $10 \mathrm{ng}$ rs-cfDNA was used as a template, which included specific parts of mutant and wild-type DNA. Digital PCR evaluated the copy number of EGFR 19 exon deletion and L858R and comparing the copy number difference of mutant genes in standard
PCR and Surveyor method. Finally, all 16 assays showed that more DNA copy numbers were detected in enriched samples (Figure 4).

\section{Surveyor Method Application to KRAS GI2X/G I 3X Screening}

Specific abnormal patterns are highly heterogeneous in individual patients, so that somatic mutations are not an ideal biomarker for cancer monitoring. ${ }^{22,23}$ About $11 \%$ of Chinese lung cancer patients have KRAS gene mutations, most located in exon 2 of G12X (G12A, G12V, G12C, G12D, G12R) and G13X (G13D, G13C). However, standard PCR detection has the advantages of convenience and high specificity. In many cases, standard PCR analysis based on probes/primers adds significant complexity to the analysis development process because probes/primers molecules for the target of interest need to be designed and tested in each assay. 

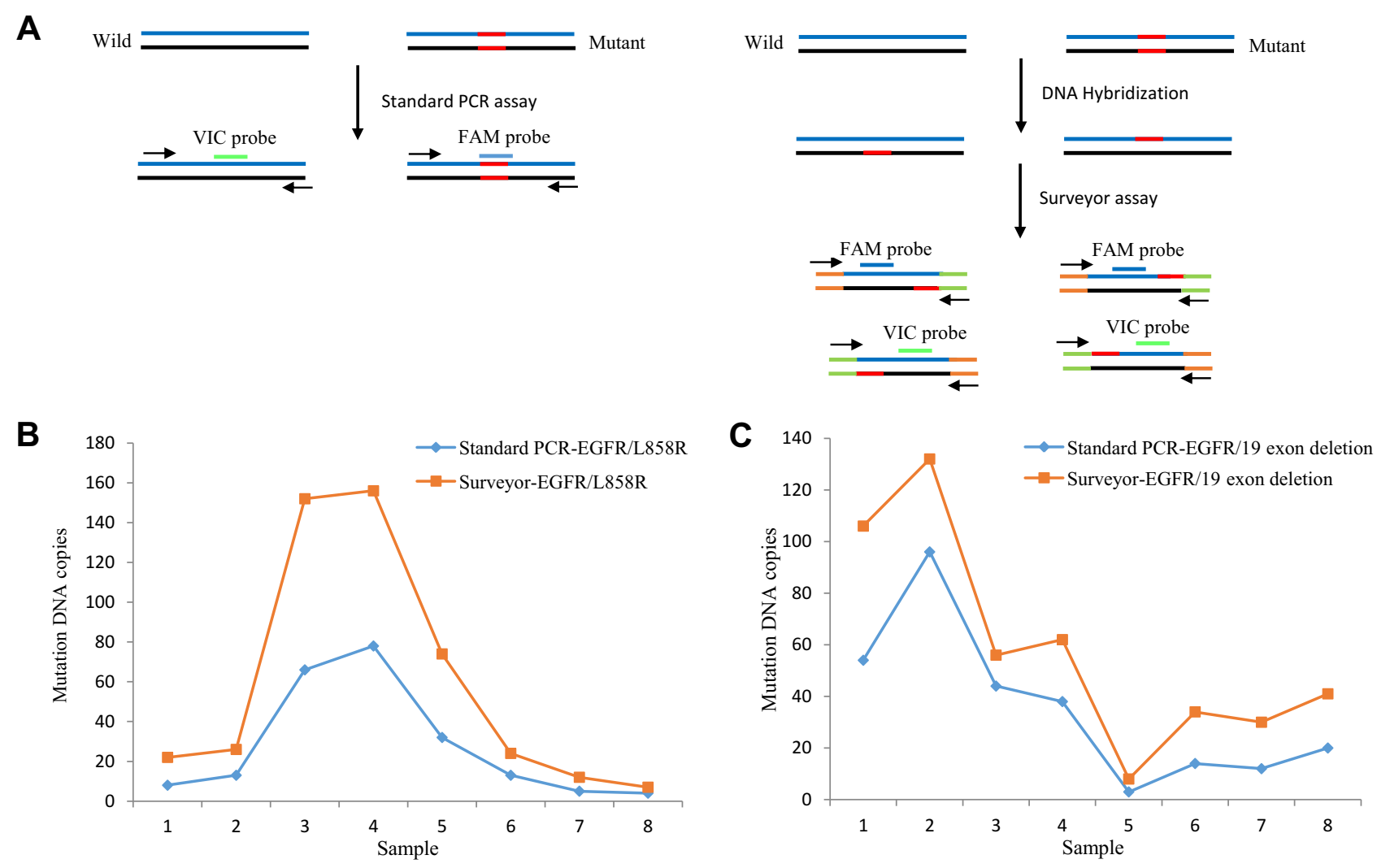

Figure 4 Compare standard PCR and Surveyor method measurement of EGFR mutant gene copy numbers across sixteen samples. (A) Schematic diagram of the standard PCR and Surveyor method assay. (B) Copy numbers detected of EGFR/L858R with standard PCR and Surveyor method. (C) Copy numbers detected of EGFR/19 exon deletion with standard PCR and Surveyor method.

In order to confirm that the Surveyor method can detect complex forms of KRAS gene mutations with the same primers and probe, we performed validation analysis on rscfDNA containing KRAS G12D and G13D. Using mutation site-specific primers and probes, the copy numbers of KRAS G12D and G13D were evaluated on 10 ng rs-cfDNA using digital PCR. Fifty-two copies of KRAS G12D (Figure 5A) and 56 copies of KRAS G13D (Figure 5B) mutation were detected. Similarly, the Surveyor method detected 82 copy number mutant genes in $10 \mathrm{ng}$ rs-cfDNA (Figure 5C). Surveyor endonuclease cuts heterogeneous hybrid DNA molecules at the position of mismatched bases, and KRAS G12D and G13D DNA fragments will be enriched together for screening with the same primer and probe. Primers are designed for the adaptors at both ends of the sequence, and the probe is designed on the normal sequence upstream of the mutant site so that two KRAS mutant genes can be detected simultaneously.

\section{The Sensitive Analysis of the Surveyor Method to Detect Mutant DNA}

The analysis of mutant genes requires a certain degree of analytical sensitivity, which usually exceeds the current technical limitations. In order to evaluate the sensitivity of the Surveyor method, rs-cfDNA containing EGFR L858R with $1 \%$ MAF will be a series of double diluted with wild-type DNA. DNA samples of MFA between $0.0078 \%$ and $1 \%$ was used as a template for sensitivity analysis, and eight replicates will perform for each assay. The Surveyor method had a limit of detection (LOD) of $0.0078 \%$ with at least $20 \mathrm{ng}$ DNA template input. Furthermore, only $0.3125 \mathrm{ng}$ DNA was needed to detect the LOD of $0.5 \%$ (Figure 6A).

We first identified Surveyor method can achieve to $0.0078 \%$ mutant fraction. We then asked whether the method could detect lower mutation frequency DNA. In order to verify that, $0.001 \%$ mutation frequency DNA was prepared by gradient dilution, and eight replicate assays were performed of the input DNA increased to $200 \mathrm{ng}$. We identified six positive results in eight independent detection experiments (Figure 6B).

\section{Discussion}

EGFR and KRAS are the most mutated oncogenes in human malignancies. In East Asia, $40-60 \%$ of patients with non-small cell lung cancer have EGFR mutations, 

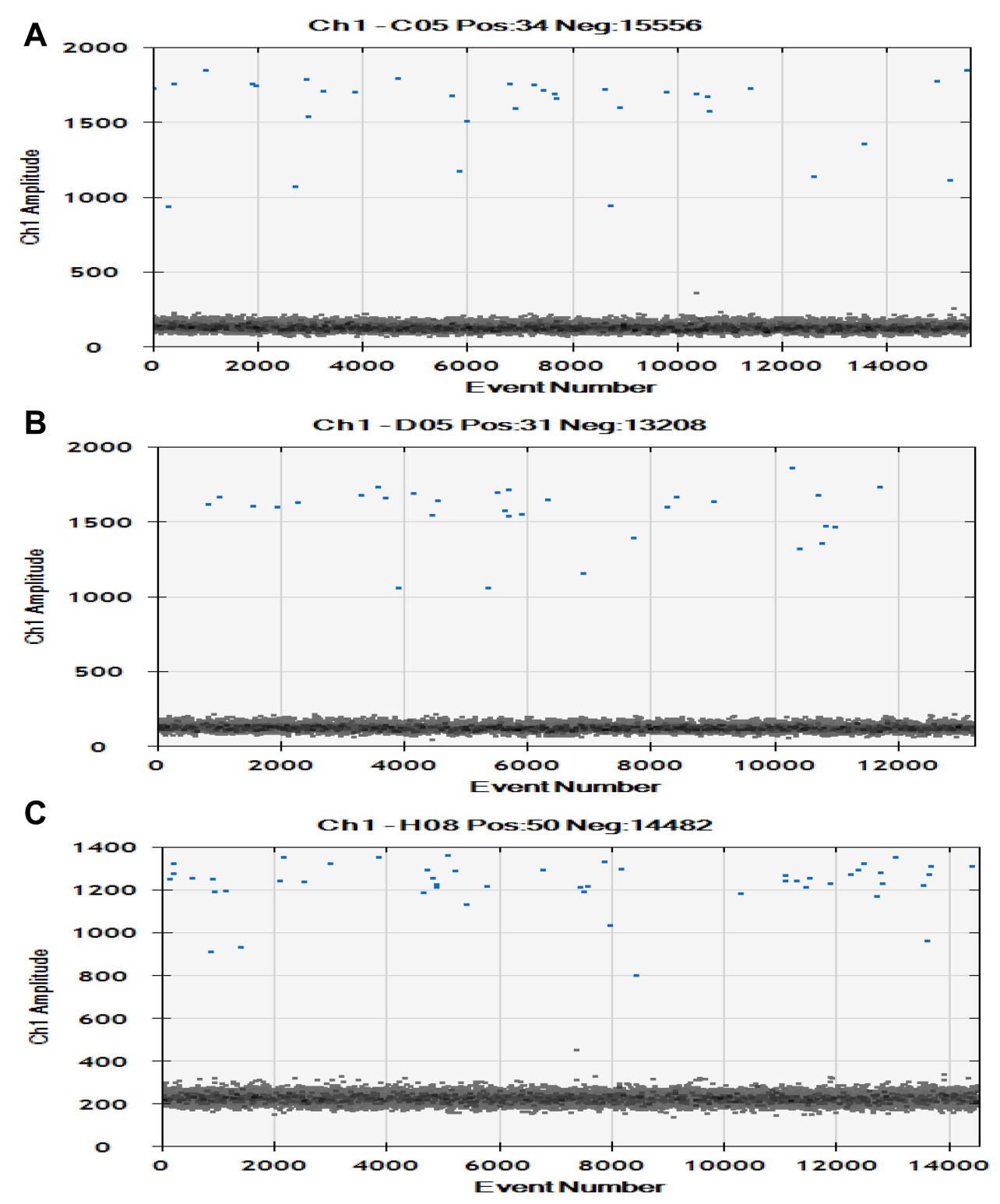

Figure 5 ddPCR measurements of KRAS GI2D/GI3D copy numbers of DNA samples. Each blue dot represents a single PCR reaction and one mutant DNA molecule. (A) Illustration of copy numbers of KRAS GI2D detected with standard PCR. (B) Illustration of copy numbers of KRAS GI3D detected with standard PCR. (C) Illustration of copy numbers of KRAS GI2D and KRAS GI3D detected with Surveyor method.

and $80 \%$ clinically respond to EGFR tyrosine kinase inhibitors gefitinib or erlotinib. ${ }^{24-28}$ Therefore, a sensitive technique is needed to identify these patients to detect the low mutation allele fraction in high background wildtype DNA in low DNA yield and low MAF samples, such as cfDNA. ${ }^{29}$ In addition, monitoring the response of cancer patients to treatment or disease progress requires repeated quantitative assessment of the disease burden.

Due to the low proportion of mutated DNA and the heterogeneity of tumor somatic mutations, standard PCR methods need to detect many tumor DNA mutation sites, making potential clinical trials too expensive and complex for all patients. ${ }^{30}$ To overcome these limitations, we developed the Surveyor method for the enrichment of mutated DNA. Enrichment increases the sensitivity of our mutant allele-specific qPCR analysis from 5\% to about $0.001 \%,{ }^{31}$ allowing the detection of very low mutant allele genomics in clinical samples positive for the two most common mutant genes.

Compared with the analytical methods proposed in other studies, ${ }^{8,9}$ the Surveyor method has advantages. The Surveyor enzyme digests the mismatched molecules; more DNA sequence information is generated for subsequent mutation DNA detection. With the same amount of DNA input, the 
A

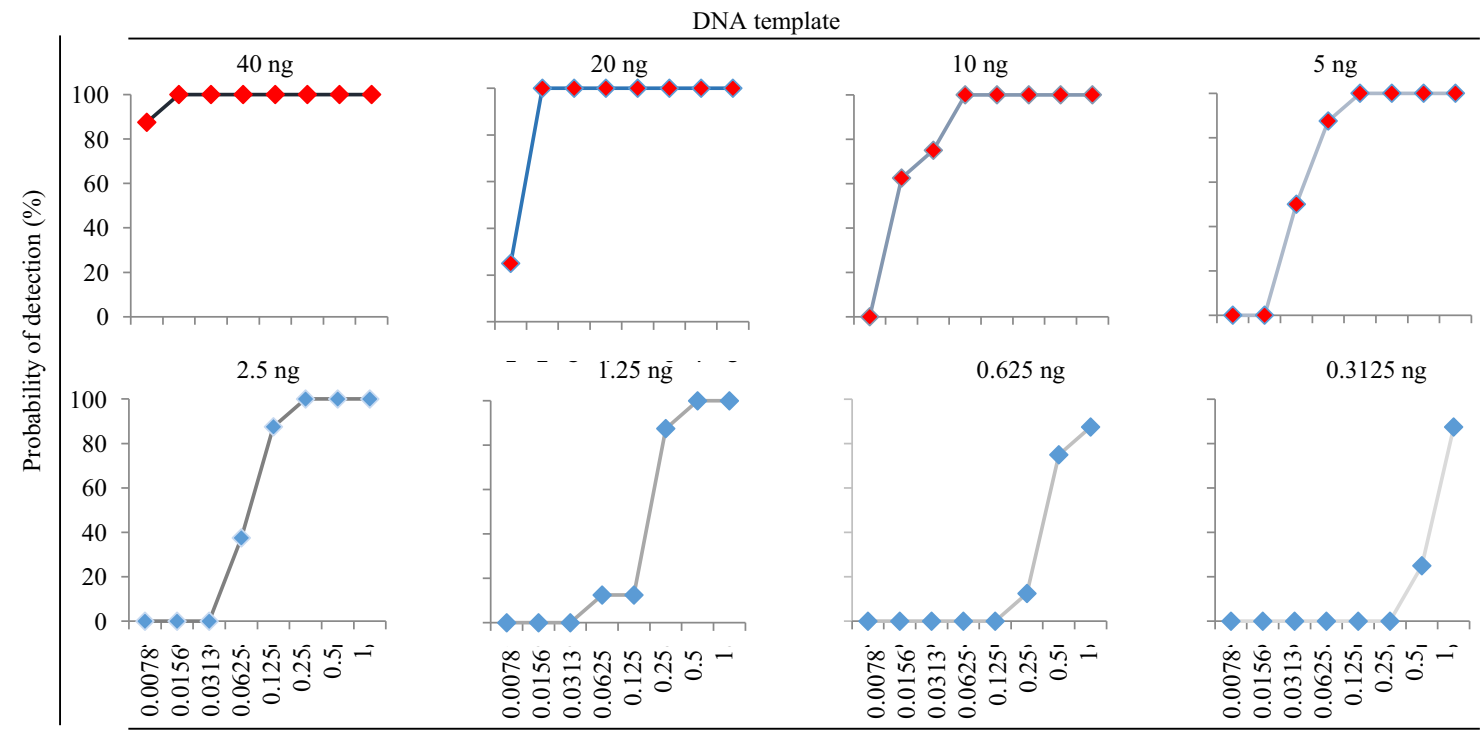

B

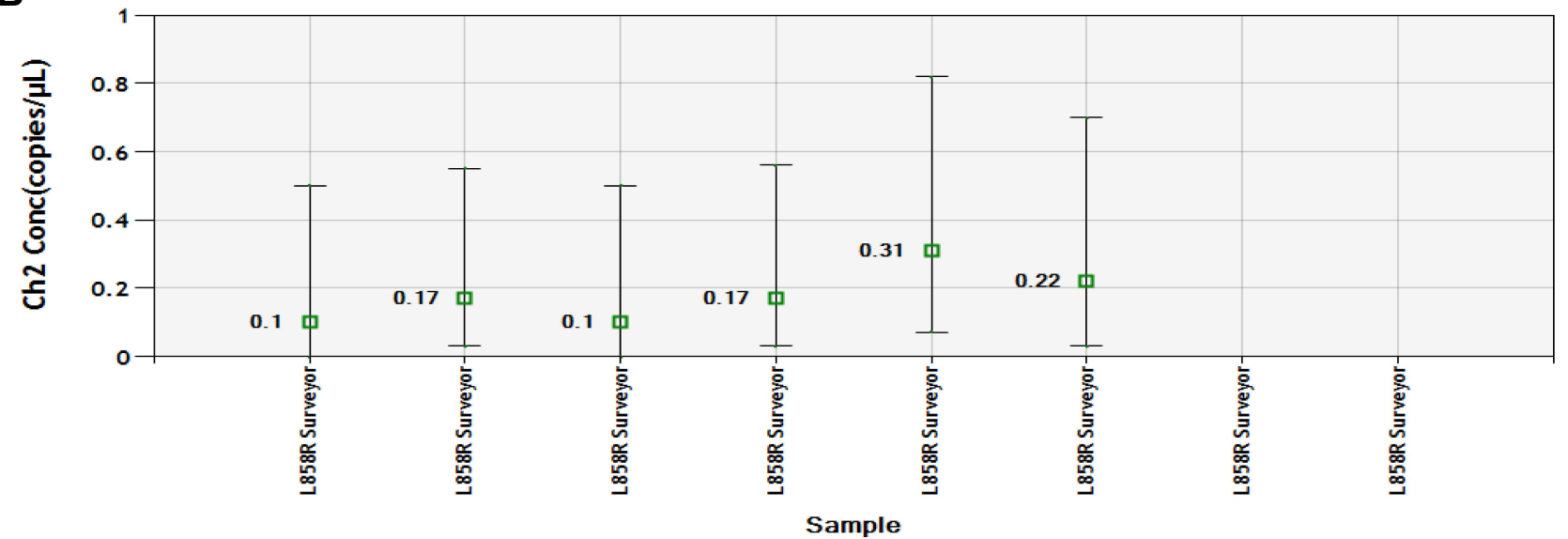

Figure 6 The Surveyor method is a sensitive approach to detect mutant DNA. (A) Serial dilution series of input DNA amount and MAF were the template for Surveyor method screening. (B) Eight technical replicates of $0.001 \%$ MAF DNA used for Surveyor method screening.

Surveyor method detected more mutant copies than the standard PCR, which will improve the sensitivity of mutant DNA detection. All PCR-based assays use fluorescent dye-labeled probe molecules to produce positive fluorescent signals. Many wild-type DNA is unnecessarily amplified in detecting single base variation, which is easy to produce false-positive signals. In many cases, the probe-based analysis adds great complexity to the development process because new probe molecules for the target of interest need to be designed and tested in each trial. The Surveyor method specifically enriches the mutant DNA sequence, and the probe is designed on the normal sequence upstream of the enzyme digestion site. This design makes the analysis and development process simpler, faster and improves the benefits of sensitivity. However, the method does not provide quantitative measurement of initial MAF before enrichment, which is meaningful in clinical monitoring.
The default may be resolved by assessing the mutation copies in per milliliter plasma.

\section{Conclusions}

We developed a simple and highly sensitive method for screening mutant genes. The Surveyor method needs to be further verified on a completely independent data set, but our study emphasizes the potential utility of mutant DNA enrichment. This method is cost-effective and sensitive in mutation sequences detection.

\section{Abbreviations}

cfDNA, cell-free DNA; MAF, Mutant Allele Frequencies; Biotin-dCTP, Biotin labelled dCTP; TdT, Terminal Transferase; rs-cfDNA, Reference Standard cfDNA; LOD, Limit of Detection. 


\section{Data Sharing Statement}

All the data and materials are available from Tables S1-S4.

\section{Acknowledgements}

We thank Jianxing He of the First Affiliated Hospital of Guangzhou Medical University for supporting the study.

\section{Author Contributions}

All authors made significant contributions to the concept, research design, implementation, data acquisition, analysis, and interpretation; participated in the drafting or critical revision of articles with important knowledge content; and finally approved the version to be submitted to the current journal and agreed to be responsible for all aspects of the work.

\section{Funding}

This study was supported by the China Postdoctoral Science Fundation (Grant 2020T130026ZX).

\section{Disclosure}

The authors declare that they have no competing interests.

\section{References}

1. Gerber DE, Gandhi L, Costa DB. Management and future directions in non-small cell lung cancer with known activating mutations. Am Soc Clin Oncol Educ Book. 2014;e353-e365. doi:10.14694/ EdBook_AM.2014.34.e353

2. Gonzalez DCD, Clarke PA, Al-Lazikani B, Workman P. Personalized cancer medicine: molecular diagnostics, predictive biomarkers, and drug resistance. Clin Pharmacol Ther. 2013;93(3):252-259. doi:10.1038/clpt.2012.237

3. Kalia M. Personalized oncology: recent advances and future challenges. Metabolism. 2013;62(Suppl 1):S11-S14. doi:10.1016/j. metabol.2012.08.016

4. Normanno N, Rachiglio AM, Roma C, et al. Molecular diagnostics and personalized medicine in oncology: challenges and opportunities. $J$ Cell Biochem. 2013;114(3):514-524. doi:10.1002/jcb.24401

5. Kim H, Ruby AE, Shandilya HG, et al. T-blocker: a simple and robust probe-free quantitative PCR assay to detect somatic mutations down to 0.1\% frequency. Biotechniques. 2018;65(4):205-210. doi:10.2144/btn2018-0111

6. Newton CR, Graham A, Heptinstall LE, et al. Analysis of any point mutation in DNA. The amplification refractory mutation system (ARMS). Nucleic Acids Res. 1989;17(7):2503-2516. doi:10.1093/nar/ 17.7.2503

7. Bolton L, Reiman A, Lucas K, Timms J, Cree IA. KRAS mutation analysis by PCR: a comparison of two methods. PLoS One. 2015;10 (1):e115672. doi:10.1371/journal.pone.0115672

8. Morlan J, Baker J, Sinicropi D. Mutation detection by real-time PCR: a simple, robust and highly selective method. PLoS One. 2009;4(2): e4584. doi:10.1371/journal.pone.0004584

9. Wang H, Jiang J, Mostert B, et al. Allele-specific, non-extendable primer blocker PCR (AS-NEPB-PCR) for DNA mutation detection in cancer. $J$ Mol Diagn. 2013;15(1):62-69. doi:10.1016/j. jmoldx.2012.08.007
10. Pender A, Garcia-Murillas I, Rana S, et al. Efficient genotyping of KRAS mutant non-small cell lung cancer using a multiplexed droplet digital PCR approach. PLoS One. 2015;10(9):e139074. doi:10.1371/ journal.pone. 0139074

11. Wood-Bouwens C, Lau BT, Handy CM, Lee H, Ji HP. Single-color digital PCR provides high-performance detection of cancer mutations from circulating DNA. $J$ Mol Diagn. 2017;19(5):697-710. doi:10.1016/j.jmoldx.2017.05.003

12. Qiu P, Shandilya H, D'Alessio JM, O'Connor K, Durocher J, Gerard GF. Mutation detection using Surveyor nuclease. Biotechniques. 2004;36(4):702-707. doi:10.2144/04364PF01

13. Huang MC, Cheong WC, Lim LS, Li MH. A simple, high sensitivity mutation screening using Ampligase mediated T7 endonuclease I and Surveyor nuclease with microfluidic capillary electrophoresis. Electrophoresis. 2012;33(5):788-796. doi:10.1002/elps.201100460

14. Tsuji T, Niida Y. Development of a simple and highly sensitive mutation screening system by enzyme mismatch cleavage with optimized conditions for standard laboratories. Electrophoresis. 2008;29 (7):1473-1483. doi:10.1002/elps.200700729

15. Wada T, Fukushima Y, Saitoh S. A new detection method for ATRX gene mutations using a mismatch-specific endonuclease. Am J Med Genet a. 2006;140(14):1519-1523. doi:10.1002/ajmg.a.31310

16. Pagniez-Mammeri H, Lombes A, Brivet M, et al. Rapid screening for nuclear genes mutations in isolated respiratory chain complex I defects. Mol Genet Metab. 2009;96(4):196-200. doi:10.1016/j. ymgme.2008.12.003

17. Jackman DM, Holmes AJ, Lindeman N, et al. Response and resistance in a non-small-cell lung cancer patient with an epidermal growth factor receptor mutation and leptomeningeal metastases treated with high-dose gefitinib. J Clin Oncol. 2006;24(27):4517-4520. doi:10.1200/JCO.2006.06.6126

18. Qiu P, Shandilya H, Gerard GF. A method for clone sequence confirmation using a mismatch-specific DNA endonuclease. Mol Biotechnol. 2005;29(1):11-18. doi:10.1385/MB:29:1:11

19. Oleykowski CA, Bronson MC, Godwin AK, Yeung AT. Mutation detection using a novel plant endonuclease. Nucleic Acids Res. 1998;26(20):4597-4602. doi:10.1093/nar/26.20.4597

20. Aggeli D, Karas VO, Sinnott-Armstrong NA, et al. Diff-seq: a high throughput sequencing-based mismatch detection assay for DNA variant enrichment and discovery. Nucleic Acids Res. 2018;46(7): e42. doi:10.1093/nar/gky022

21. Wan JCM, Heider K, Gale D, et al. CtDNA monitoring using patient-specific sequencing and integration of variant reads. Sci Transl Med. 2020;12(548):z8084. doi:10.1126/scitranslmed. aaz8084

22. Luo H, Zhao Q, Wei W, et al. Circulating tumor DNA methylation profiles enable early diagnosis, prognosis prediction, and screening for colorectal cancer. Sci Transl Med. 2020;12(524):x7533. doi:10.1126/scitranslmed.aax7533

23. Kandoth C, Mclellan MD, Vandin F, et al. Mutational landscape and significance across 12 major cancer types. Nature. 2013;502 (7471):333-339. doi:10.1038/nature12634

24. Sequist LV, Bell DW, Lynch TJ, Haber DA. Molecular predictors of response to epidermal growth factor receptor antagonists in non-small-cell lung cancer. J Clin Oncol. 2007;25(5):587-595. doi:10.1200/JCO.2006.07.3585

25. Paez JG, Janne PA, Lee JC, et al. EGFR mutations in lung cancer: correlation with clinical response to gefitinib therapy. Science. 2004;304(5676):1497-1500. doi:10.1126/science.1099314

26. Lynch TJ, Bell DW, Sordella R, et al. Activating mutations in the epidermal growth factor receptor underlying responsiveness of non-small-cell lung cancer to gefitinib. $N$ Engl J Med. 2004;350 (21):2129-2139. doi:10.1056/NEJMoa040938 
27. Pao W, Miller V, Zakowski M, et al. EGF receptor gene mutations are common in lung cancers from "never smokers" and are associated with sensitivity of tumors to gefitinib and erlotinib. Proc Natl Acad Sci USA. 2004;101(36):13306-13311. doi:10.1073/pnas.0405220101

28. Shigematsu H, Lin L, Takahashi T, et al. Clinical and biological features associated with epidermal growth factor receptor gene mutations in lung cancers. J Natl Cancer Inst. 2005;97(5):339-346. doi:10.1093/jnci/dji055

29. Keraite I, Alvarez-Garcia V, Garcia-Murillas I, et al. PIK3CA mutation enrichment and quantitation from blood and tissue. Sci Rep. 2020;10(1). doi:10.1038/s41598-020-74086-w
30. Jin S, Zhu D, Shao F, et al. Efficient detection and post-surgical monitoring of colon cancer with a multi-marker DNA methylation liquid biopsy. Proc Natl Acad Sci USA. 2021;118(5):e2017421118. doi:10.1073/pnas.2017421118

31. Alvarez-Garcia V, Bartos C, Keraite I, et al. A simple and robust real-time qPCR method for the detection of PIK3CA mutations. Sci Rep. 2018;8(1):4290. doi:10.1038/s41598-018-22473-9

\section{Publish your work in this journal}

Cancer Management and Research is an international, peer-reviewed open access journal focusing on cancer research and the optimal use of preventative and integrated treatment interventions to achieve improved outcomes, enhanced survival and quality of life for the cancer patient.
The manuscript management system is completely online and includes a very quick and fair peer-review system, which is all easy to use. Visit http://www.dovepress.com/testimonials.php to read real quotes from published authors. 